\title{
Benign Childhood Central Nervous System Neoplasm
}

National Cancer Institute

\section{Source}

National Cancer Institute. Benign Childhood Central Nervous System Neoplasm. NCI

Thesaurus. Code C5591.

A non-metastasizing neoplasm that arises from the central nervous system during

childhood. 